\title{
Technique for Determining the Fuel Consumption of a Vehicle on a Given Route
}

\author{
Vladislav Kravets ${ }^{\mathrm{a}}$, Roman Musarsky ${ }^{\mathrm{b}}$, Anton Tumasov ${ }^{\mathrm{c}}$ \\ Transport Systems Institute, Nizhny Novgorod State Technical University n.a. R.E. Alekseev (NNSTU), Nizhny Novgorod, \\ Russian Federation \\ aait@nntu.nnov.ru, ${ }^{b}$ musarsky@list.ru, ${ }^{c}$ anton.tumasov@gmail.com
}

\begin{abstract}
The article presents the results of calculation and experimental determination of the fuel consumption of light commercial vehicle on a given country route traffic as well as structure of methodology of fuel consumption estimation.
\end{abstract}

Keywords-fuel consumption, light commercial vehicle, traffic and route characteristics, vehicle dynamic characteristics, fueleconomic characteristics

\section{INTRODUCTION}

The purpose of this study is theoretical and experimental determination of fuel consumption of light commercial vehicle (LCV) on a given country route traffic. The object of the study is LCV of Russian manufacturer GAZelle «Next», relating to vehicles of category $\mathrm{N} 1$. Constructive parameters of the test vehicle are presented by the authors [1].

Fuel economy of the vehicle and the associated environmental protection very often attracts the attention of automobile engineers $[2,3]$. The basic analytical technique of the calculation of fuel consumption when the vehicle is operated on a given route by means of graph definition proposed by G.V. Zimelev and described in [4]. Graph analytical determination of fuel consumption is performed by using:

1) The characteristics of the travel route;

2) The dynamic characteristics of the vehicle;

3) Fuel-economic characteristics of the vehicle.

This paper presents the structure of methodology of fuel consumption prediction that is based on known theoretical equations with some practical adjustment and proved by experimental research made in real road traffic.

\section{THEORETICAL BASICS OF RESEARCH}

A description of the travel route is a set of road sections with a constant throughout the length of resistance coefficient of the road. The parameters of the test route Nizhny Novgorod - Pavlovo (country road in Nizhny Novgorod region - 71,3 km length) was determined by processing the satellite data by the method described in [1], according to the GLONASS / GPS system measured length of individual sections of the route, with constant values of longitudinal slopes. The measurement results are shown on Fig. 1.

For the description of characteristics of the route for each value of the longitudinal slope of the road several parameters were calculated:
Longitudinal slope angle . $\operatorname{arctg}(i)$ and resistance coefficient of the road $=f \cos . \pm \sin$. where, $f=0,012-$ the coefficient of rolling resistance [4];

Longitudinal slope angle of the road. The "+" - is on the rise, "-" - on the way down. For $i=0 ; 1 ; 2 ; 3 ; 4 \%$ of $=0,012$; $0.022 ; 0.032 ; 0.042 ; 0.052$.

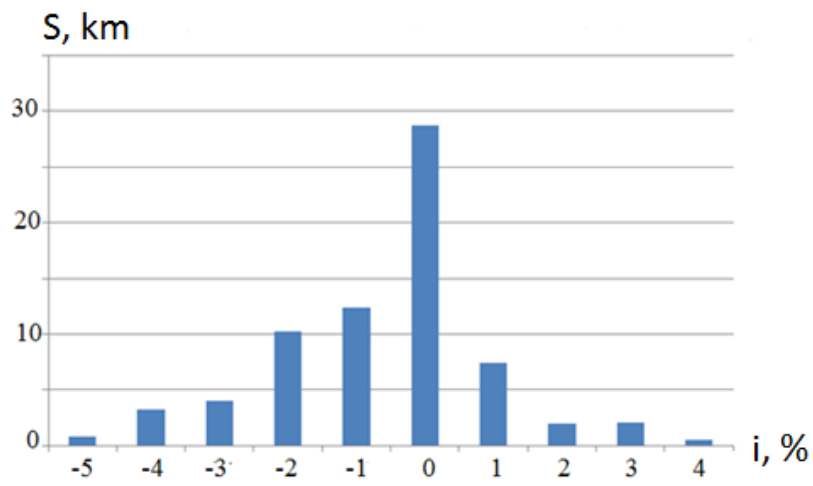

Figure 1. Distribution of length sections of the route, depending on the values of their longitudinal slope.

In accordance with the procedure described in [4], computed sequentially:

The dynamic characteristics of LCV;

The fuel-economic characteristics of the steady motion of a vehicle.

The dynamic characteristic of LCV is the dependence between the dynamic factor and the speed of movement for each gear of transmission (Fig. 2). This diagram has also the numerical values of the resistance coefficient of the road for all values of longitudinal slope, taken from Figure 1. At the points of intersection of curves it is possible to define the maximum vehicle speed It follows that for a given route, having a relatively small slope not exceeding $4 \%$, LCV has to move on highest (5th) transmission gearbox, the maximum dynamic factor which is equal to 0,07 .

Fuel-economic characteristics of the steady motion of a vehicle are dependent on the speed steady motion on roads with different coefficients of resistance. Specific fuel consumption, depending on the speed limit and the engine load is calculated by the approximate analytical method developed by I.S. Schlippe [4]. Correction factors calculated by the analytical dependence described in [4] and [5]. The power of resistance of the road is calculated at values = 0,$012 ; 0,022 ; 0,032 ; 0,042 ; 0,052$. The fuel-economic 
characteristic of the LCV when driving on highest (5th) transmission gearbox is shown on Fig. 3.

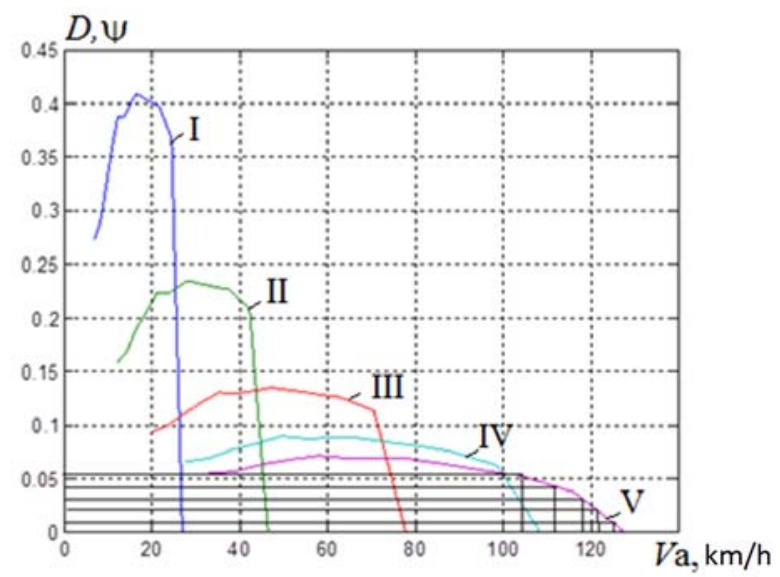

Figure 2. LCV dynamic characteristics.

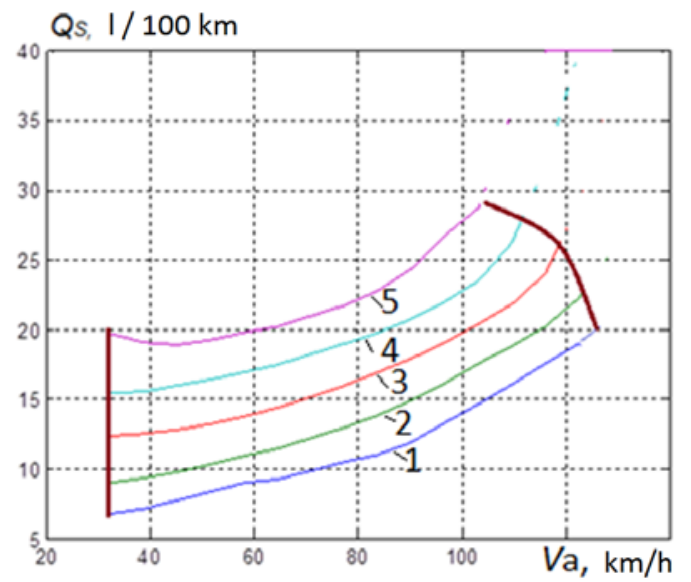

Figure 3. The fuel-economic characteristics of the LCV when driving on highest (5th) transmission gearbox at the resistance coefficient of the road : $1-0,012 ; 2-0,022 ; 3-0,032 ; 4-0,042 ; 5-0,052$.

The above characteristics were used to calculate the fuel consumption for a given route by means of graph-analytical method. On the diagram (Fig. 4) fuel-economic characteristics put in the right lower quadrant, dynamic performance - in the right upper quadrant, the characterization of the route - in the upper left quadrant, with values in the upper quadrants are applied to the same scale. Calculations are made on the assumption that each section of the road with a constant coefficient of resistance of the vehicle is moving as fast as possible.

Area in the lower left quadrant of an appropriate scale is equivalent fuel consumption in each section of the route:

$$
Q_{j}=\frac{Q_{S j} S_{j}}{100}
$$

Where Qsj - track fuel consumption on $j$ - section of the route $(1 / 100 \mathrm{~km}) ; S j$ - length of the $j$-leg, km. Total fuel consumption on the route is the sum of the consumption of fuel on each section of the route:

$$
Q=\sum_{j=1}^{n} Q_{S j}
$$

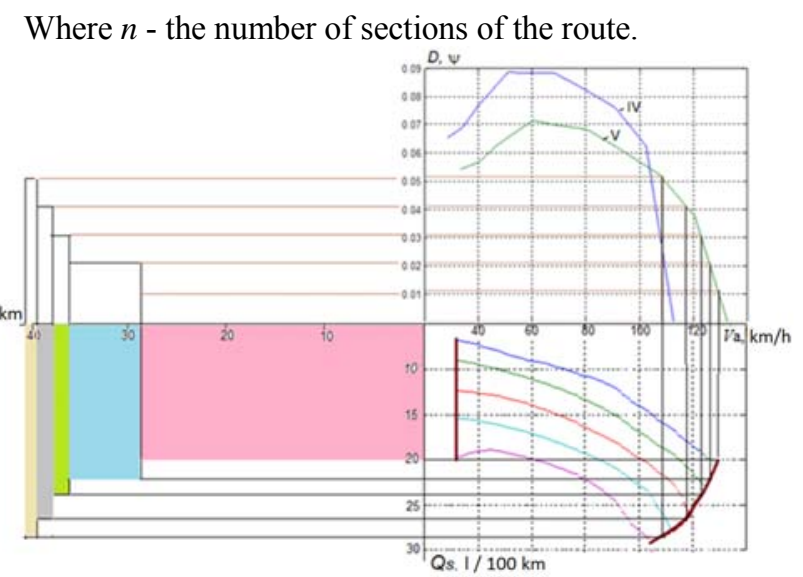

Figure 4. Scheme of determining fuel consumption for a given route.

Calculations with using of a scheme shown on Fig. 4 reveal that the total fuel consumption of LCV on the route Nizhny Novgorod - Pavlovo length 71,3 km was 8,30 liters. Fuel consumption was taken into account only when the vehicle is moving along horizontal parts of the road and on the rise.

\section{EXPERIMENTAL RESEARCH}

To verify the calculated data the road tests were carried out. Preparation of LCV to the road test was performed in accordance with the requirements set out in the regulations $[6,7]$. Tests were carried out with the full weight of the car $M a=3500 \mathrm{~kg}$.

During the tests, the following parameters were recorded: total mileage, mileage on the site, altitude, the slope of the road, the number and length at the site, the fuel consumption at the site, the total overall consumption, vehicle speed, the overall total mileage, travel time, position of pedals (clutch and brake), fuel checking idling air mass flow rate, the instantaneous fuel efficiency, a transmission gear ratio, the degree of capacity utilization and engine speed.

While driving along the route the multifunction measurement equipment of NNSTU Center "Transport systems" was used: GLONASS / GPS system Racelogic; fuel consumption meter Kistler Automotive $\mathrm{GmbH}$; the collecting data device Tokyo Sokki Kenkyujo with CAN module and CUMMINS diagnostic equipment. All measured parameters were recorded on a personal computer GETAC. The process of testing was fixed on the car DVR SHO-ME. Communication control unit CUMMINS carried out with a personal computer via the CAN bus. Using of modern measurement equipment allowed record all parameters with high accuracy.

The experimental fuel consumption was 8,36 liters. 


\section{KEY RESULTS AND CONCLUSIONS}

1. Using of GLONASS / GPS system data allowed receiving characteristic of the route of the vehicle that was necessary for relatively precise calculating of fuel consumption.

2. The theoretically estimated value of LCV fuel consumption of the test route Nizhny Novgorod - Pavlovo was 8,3 liters. The experimental fuel consumption on a given route that was measured during LCV road test was 8,36 liters. High convergence of the results of theoretical and experimental determination of the fuel consumption for a given route suggests the possibility of using graphicanalytical methods, presented in the paper, for approximate calculations (predictions) of the fuel economy of new vehicles (including LCV).

3. Further development of the methodology of calculation of fuel consumption for a given route traffic should be conducted in the following areas:

- Take into account the fuel consumption during acceleration or deceleration of a vehicle;

- Take into account the distribution of velocities that are not constant;

- Take into account complex transmission behavior;

- Take into account the experimentally determined values of the coefficients of rolling resistance of vehicles wheels for a real bearing surface sections of the route of movement.

\section{ACKNOWLEDGMENT}

The research was done with the financial support of Ministry of Education and Science of the Russian Federation in the frame of the complex project "The establishment of the advanced technology production of ecological and resources-economy LCV" under the contract №02.G25.31.006 from 12.02.2013 (Governmental Regulation №218 from 09.04.2010).

\section{REFERENCES}

[1] Kravets V.N., Musarsky R.A. Influence of macro road profile on fuel consumption of the truck // Proceedings of the NSTU n.a. R.E. Alekseev. - 2014. - № 4 (106). - P. 196 - 200. http://www.nntu.ru/trudy/2014/04/196-200.pdf

[2] M. G. Lee, Y. K. Park, K. K. Jung and J. J. Yoo, "Estimation of Fuel Consumption using In-Vehicle Parameters", International Journal of $\mathrm{u}$ - and e- Service, Science and Technology, vol. 4, No. 4, December, 2011,pp. 37-46

[3] Y. Saboohia, H. Farzanehb, "Model for developing an eco-driving strategy of a passenger vehicle based on the least fuel consumption," Applied Energy, vol. 86, issue 10, 2009, pp. 1925-1932.

[4] Kravets V.N., Selifonov V.V. The theory of the car. - M .: OOO "Greenlight +", 2011. - 884 P.

[5] Grishkevich A.I. Cars theory. - Minsk: Higher School, 1986 - 208 P.

[6] GOST R 54810-2011. Automotive vehicles. Fuel economy. Test methods. - Enter. 2012-09-01. - M.: Federal Agency for Technical Regulation and Metrology: Standartinform, 2012.

[7] UNECE R84 - measurement of fuel consumption. 